\title{
Efficacy and Safety of Thrombolytic Therapy in Acute Submassive Pulmonary Embolism: Follow-Up Study
}

\author{
Santosh Kumar Sinha, ${ }^{a}$, Mohit Sachan ${ }^{\mathrm{a}}$, Amit Goel ${ }^{\mathrm{a}}$, Karandeep Singh ${ }^{\mathrm{a}}$, Vikas Mishra ${ }^{\mathrm{a}}$, \\ Mukesh Jitendra Jha ${ }^{\mathrm{a}}$, Ashutosh Kumar ${ }^{\mathrm{a}}$, Nasar Abdalia, Mohammad Asifa, Mahamdula Razi ${ }^{\mathrm{a}}$, \\ Umeshwar Pandey ${ }^{\mathrm{a}}$, Ramesh Thakur ${ }^{\mathrm{a}}$, Chandra Mohan Varma ${ }^{\mathrm{a}}$, Vinay Krishna ${ }^{\mathrm{a}}$
}

\begin{abstract}
Background: Thrombolysis in acute submassive pulmonary embolism (PE) remains controversial. So we studied impact of thrombolytic therapy in acute submassive PE in terms of mortality, hemodynamic status, improvement in right ventricular function, and safety in terms of major and minor bleeding.
\end{abstract}

Method: A single-center, prospective, randomized study of 86 patients was conducted at LPS Institute of Cardiology, G.S.V.M. Medical College, Kanpur, India. Patients received thrombolysis (single bolus of tenecteplase) with unfractionated heparin (UFH, group I) or placebo with UFH (group II).

Result: Mean age of patients was $54.35 \pm 12.8$ years with male dominance (M:F $=70 \%: 30 \%)$. Smoking was the most common risk factor seen in $29 \%$ of all patients, followed by recent history of immobilization $(25 \%)$, history of surgery or major trauma within past 1 month $(15 \%)$, dyslipidemia (10\%) and diabetes mellitus (10\%). Dyspnea was the most common symptom in $80 \%$ of all patients, followed by chest pain in $55 \%$ and syncope in $6 \%$. Primary efficacy outcome occurred significantly better in group I vs. group II ( $4.5 \%$ vs. $20 \%$; P = 0.04$)$, and significant difference was also found in hemodynamic decompensation $(4.5 \%$ vs. $20 \%$; $\mathrm{P}=0.04)$, the fall in mean pulmonary artery systolic pressure (PASP) $(28.8 \%$ vs. $22.5 \% ; \mathrm{P}=0.03)$, improvement in right ventricular $(\mathrm{RV})$ function $(70 \%$ vs. $40 \% ; \mathrm{P}=0.001)$ and mean hospital stay $(8.1 \pm 2.5$ vs. $11.1 \pm 2.14$ days; $\mathrm{P}=0.001)$. There was no difference in mortality and major bleeding as safety outcome but increased minor bleeding occurred in group I patients $(16 \%$ vs. $12 \%$; $\mathrm{P}=0.04)$.

Conclusion: Patients with acute submassive PE do not derive overall mortality benefit, recurrent $\mathrm{PE}$ and rehospitalization with thrombolytic therapy but had improved clinical outcome in form of decrease in hemodynamic decompensation, mean hospital stay, PASP and improvement of RV function with similar risk of major bleed but at cost

Manuscript accepted for publication November 28, 2016

aDepartment of Cardiology, LPS Institute of Cardiology, G.S.V.M. Medical College, Kanpur, Uttar Pradesh 208002, India

${ }^{b}$ Corresponding Author: Santosh Kumar Sinha, Department of Cardiology, LPS Institute of Cardiology, G.S.V.M. Medical College, Kanpur, Uttar Pradesh 208002, India. Email: fionasan@rediffmail.com

doi: https://doi.org/10.14740/jocmr2829w of increased minor bleeding.

Keywords: Thrombolysis; Submassive pulmonary embolism; Tenecteplase; Hemodynamic decompensation; Right ventricular function; Pulmonary artery systolic pressure

\section{Introduction}

Acute pulmonary embolism (PE) is the most serious clinical presentation of venous thromboembolism (VTE). VTE is associated with a mortality rate exceeding $15 \%$ in first 3 months after diagnosis [1] with survivors remaining at risk for development of recurrent PE and/or chronic thromboembolic pulmonary hypertension [2]. The clinical benefits of thrombolysis have remained controversial in the absence of hemodynamic compromise at presentation, i.e., submassive PE.

Pulmonary emboli most often arise from the deep veins of the lower extremity and pelvis from where they dislodge to the pulmonary arteries causing abnormalities in hemodynamics and gas exchange depending on the size of the embolus, the patient's underlying cardiopulmonary status, and compensatory neurohumoral adaptations. In addition to physical obstruction, acute PE leads to the release of pulmonary artery vasoconstrictors and hypoxemia, with a subsequent increase in pulmonary vascular resistance and right ventricular (RV) afterload, dilatation, hypokinesia, tricuspid regurgitation, RV failure leading to a reduction of the cardiac output causing systemic hypotension and hemodynamic instability [3, 4]. The association between elevated levels of biomarkers of myocardial injury and an adverse early outcome indicates that RV ischemia is of pathophysiological significance in the acute phase of PE [5, 6]. Acute PE has been classified into three groups: non-massive or low risk, submassive or moderate/intermediate risk, and massive or high risk. Massive PE is defined as conglomerate of pulselessness, persistent bradycardia with rate $<40 \mathrm{bpm}$ and signs of shock or sustained hypotension. Sustained hypotension includes systolic blood pressure (SBP) of $<90 \mathrm{~mm} \mathrm{Hg}>15 \mathrm{~min}$, an SBP of $<100$ $\mathrm{mm} \mathrm{Hg}$ in a patient with a history of hypertension, or a $>40 \%$ reduction in baseline SBP in absence of dysrhythmia, hypovolemia, sepsis, or left ventricular (LV) dysfunction [7]. Submassive PE is one with normal or near normal SBP $(\geq 90 \mathrm{~mm} \mathrm{Hg})$ with evidence of cardiopulmonary stress, including RV dysfunc- 


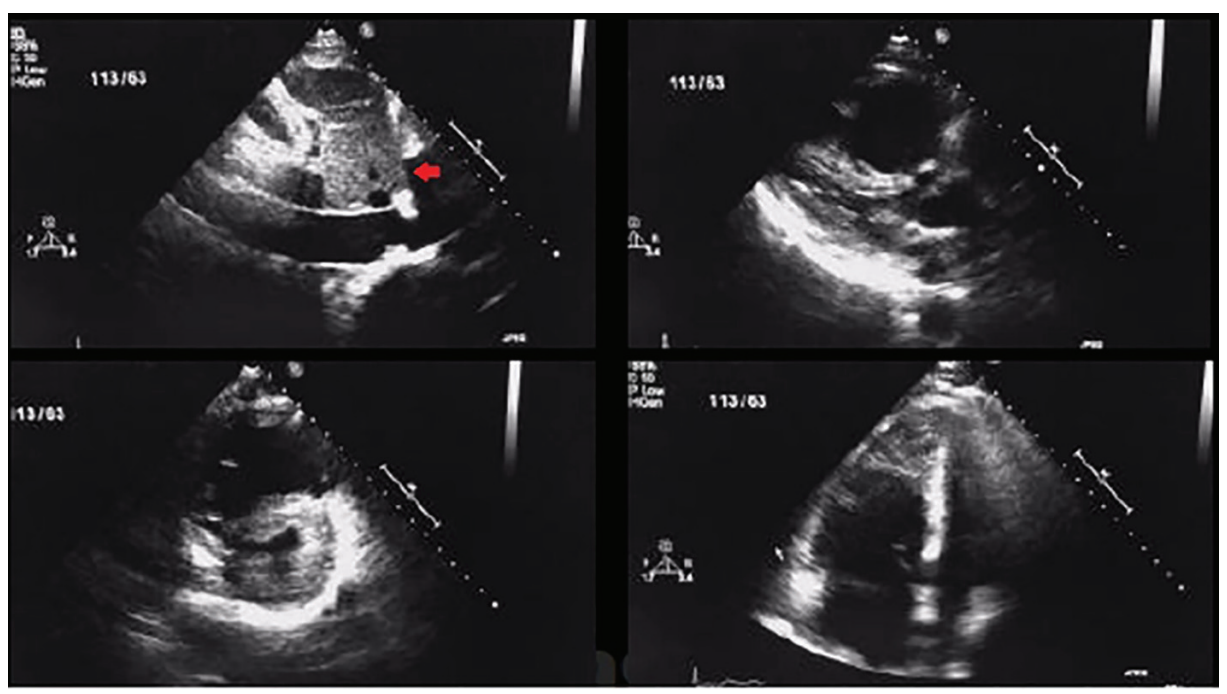

Figure 1. Transthoracic 2D echocardiogram images showing RV dilatation and dysfunction along with thrombus in a patient with acute pulmonary embolism (arrow showing thrombus in short axis view).

tion or myocardial necrosis. RV dysfunction is defined by RV dilation on echo (RV diameter/LV diameter $>0.9)$, RV systolic dysfunction on echo, brain natriuretic peptide (BNP) elevation $(>90 \mathrm{pg} / \mathrm{mL}), \mathrm{N}$-terminal pro-BNP $(>500 \mathrm{pg} / \mathrm{mL})$ or electrocardiographic (ECG) changes (new RBBB, anteroseptal ST $\uparrow / \downarrow$, or anteroseptal T-wave $\downarrow$ ). Myocardial necrosis is defined as elevation in troponin I or T over laboratory normal value or above patient baseline [7]. Non-massive PE is defined as no signs of clinical instability, hemodynamic compromise, or RV strain depending on echocardiographic finding or biomarker [7].

\section{Materials and Methods}

This was a prospective, randomized, single-center study conducted in the Department of Cardiology, LPS Institute of Cardiology, G.S.V.M. Medical College, Kanpur, UP, India between January 2012 and July 2015. Eighty-seven consecutive patients of age 18 - 75 years with acute PE (symptoms $\leq 14$ days before presentation) were enrolled with evidence of right ventricular dysfunction as diagnosed by echocardiography (Fig. 1) and/or biochemical evidence of myocardial injury and confirmed by contrast-enhanced computed tomography pulmonary angiogram (CECT) (Fig. 2). Exclusion criteria were 1) symptoms $\geq 14$ days; 2 ) age $\leq 18$ years or $\geq 75$ years; 3 ) known hypersensitivity to streptokinase, tenecteplase (TNK) or heparin; 4) uncontrolled hypertension (SBP $>180 \mathrm{~mm}$ $\mathrm{Hg}$ and/or diastolic blood pressure (DBP) $>110 \mathrm{~mm} \mathrm{Hg}$ at presentation; 5) $\mathrm{SBP}<90 \mathrm{~mm} \mathrm{Hg}$ for more than $15 \mathrm{~min}$ at presentation; 6) known coagulation disorder (including use of vitamin $\mathrm{K}$ antagonist), platelet count $<100,000 / \mathrm{mm}^{3}$; and 7) known intracranial neoplasm, past history of hemorrhagic stroke, ischemic stroke within 1 year, active peptic ulcer or active bleeding (except menstruation). The study protocol was approved by the local ethics committee and followed the Declaration of Helsinki after obtaining informed consent from each patient.

\section{Procedure}

Enrolled patients were assessed for risk factors and underwent comprehensive evaluation including detailed clinical examination, hospital course and investigations including ECG, cardiac enzymes (troponin $\mathrm{T}$ and troponin $\mathrm{I}$ ), 2D echocardiogram by a VIVID [6] (GE Healthcare) instrument and CT pulmonary angiography as a part of their diagnostic procedure. Echocardiographic examination was repeated at day 7 (D7) for assessment of pulmonary artery systolic pressures (PASPs) and RV function.

Hemodynamic stability was defined as $\mathrm{SBP} \geq 90 \mathrm{~mm} \mathrm{Hg}$ without need of any ionotropic support. RV dysfunction was defined as RV dilation (apical four-chamber RV diameter divided by LV diameter $>0.9$ ) and/or RV systolic dysfunction by tricuspid annular planar systolic excursion (TAPSE) $<16 \mathrm{~mm}$ as measured in apical four-chamber view. Myocardial injury was defined as elevation of troponin I ( $>0.4 \mathrm{ng} / \mathrm{mL})$ or troponin $\mathrm{T}(>0.1 \mathrm{ng} / \mathrm{mL})$.

Eligible patients were randomly allocated into two groups within $1 \mathrm{~h}$ after diagnosis. Group I $(\mathrm{n}=45)$ received fibrinolysis in form of single weight adjusted bolus dose of intravenous TNK over a period of $5-10 \mathrm{~s}$ and group II $(\mathrm{n}=41)$ received same volume of placebo (normal saline). Unfractionated heparin (UFH) was administered in both groups: group I received only infusion while group II received initial bolus followed by infusion to maintain an activated partial thromboplastin time (APTT) 2.0 - 2.5 times the upper limit of the normal range. All patients were discharged provided they were asymptomatic, $\mathrm{SBP}>100 \mathrm{~mm} \mathrm{Hg}$ and no active bleeding.

\section{Follow-up and outcome assessment}

The primary efficacy outcome was clinical composite of death from any cause, hemodynamic decompensation or collapse 


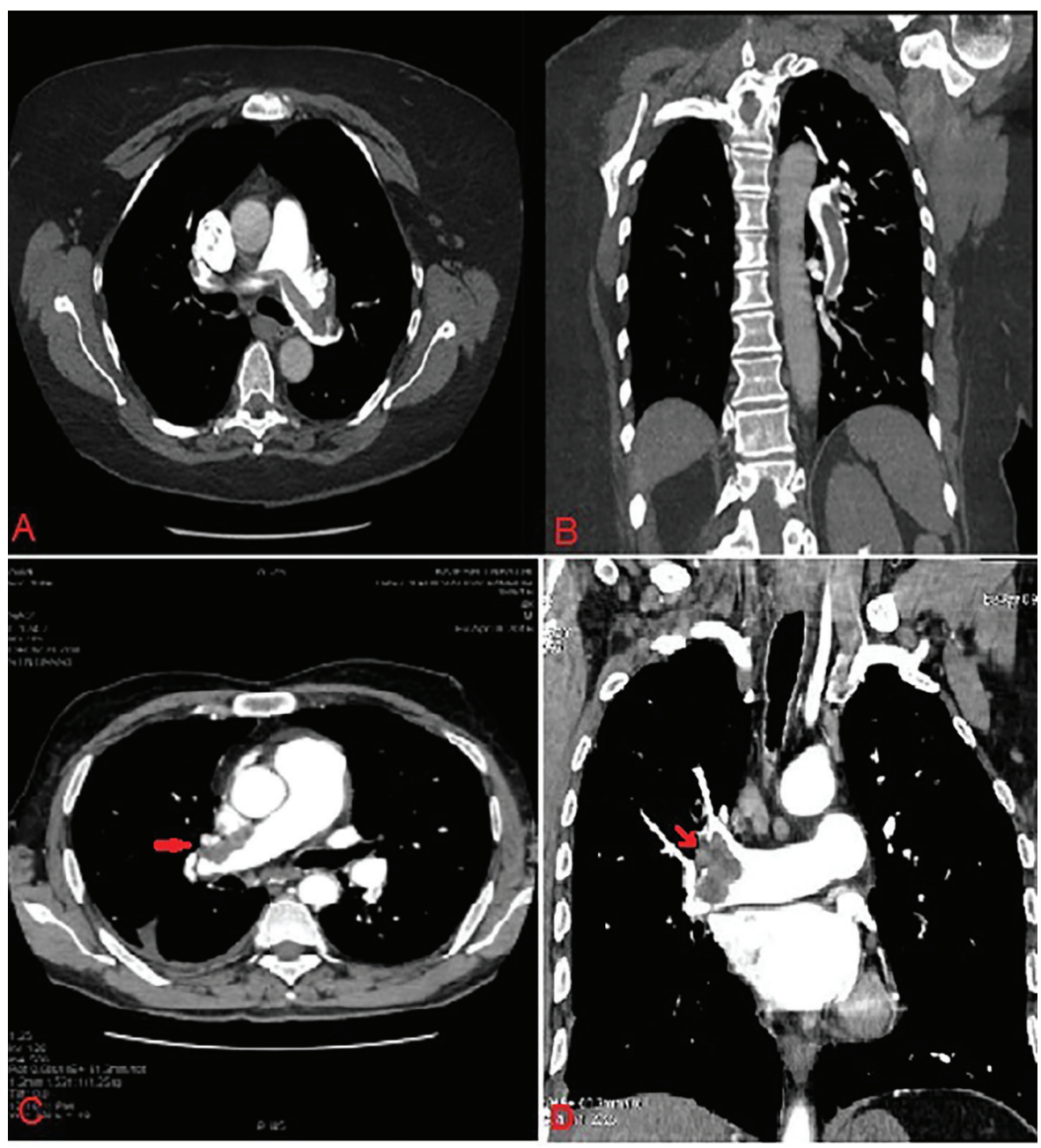

Figure 2. Contrast-enhanced computed tomography of pulmonary vasculature showing large saddle thrombus in right pulmonary artery (red arrow).

within 7 days after randomization. The secondary outcomes were composite of primary outcome and symptomatic recurrence of $\mathrm{PE}$ within 7 days, death and rehospitalization within 30 days. Safety outcomes were defined as ischemic or hemorrhagic stroke (including hemorrhagic conversion of ischemic stroke) within 7 days after randomization, extracranial major bleeding within 7 days according to The International Society on Thrombosis and Haemostasis (ISTH) definition [6]. Hemodynamic decompensation was defined by need for cardiopulmonary resuscitation, $\mathrm{SBP}<90 \mathrm{~mm} \mathrm{Hg}$ for $\geq 15 \mathrm{~min}$, drop in SBP by $\geq 40 \mathrm{~mm} \mathrm{Hg}$ for $\geq 15 \mathrm{~min}$ with findings of end-organ hypoperfusion, or need for vasopressors.

\section{Statistical analysis}

Statistical analyses were performed using the SPSS 17.0 (SPSS Inc., Chicago, IL, USA). Continuous variables were expressed as mean \pm standard deviation, whereas categorical variables were given as numbers (percentages). The comparison between groups was done by Mann-Whitney U-test, Student's $t$-test for continuous variables and by Chi-square or Fisher's exact test for categorical variables. $\mathrm{P}<0.05$ was considered statistically significant.

\section{Results}

\section{Baseline characteristics}

Mean age of patients $(n=45)$ who received thrombolysis with TNK was $54.35 \pm 12.1$ years which was comparable with the age in placebo group $55.12 \pm 11.7$ years. All patients in both groups were normotensives at presentation, with mean SBP in group I being $111.0 \pm 9.79 \mathrm{~mm} \mathrm{Hg}$ while $112.1 \pm 10.02$ $\mathrm{mm} \mathrm{Hg}$ in group II. Mean duration of illness before presentation in the study population was $3.49 \pm 1.97$ days which was $3.65 \pm 2.25$ days in group I and $3.3 \pm 1.83$ days in group II $(\mathrm{P}=0.6)$. Smoking was the most common risk factor for $\mathrm{PE}$ which was present in $29 \%(n=25)$ of all the patients, followed by recent history of immobilization in $25 \%(n=22)$, 
Table 1. Baseline Clinical Characteristics of the Study Patients in Both Groups $(n=86)$

\begin{tabular}{|c|c|c|c|}
\hline Variables & Group I (TNK; $n=45)$ & Group II (placebo; $n=41)$ & P-value \\
\hline Age (years) & $54.35 \pm 12.1$ & $55.12 \pm 11.7$ & 0.2 \\
\hline $\operatorname{Sex}(M: F)$ & $31 / 14$ & $29 / 12$ & 0.4 \\
\hline BMI $\left(\mathrm{kg} / \mathrm{m}^{2}\right)$ & $26.5 \pm 4.8$ & $27.35 \pm 3.7$ & 0.13 \\
\hline $\mathrm{SBP}(\mathrm{mm} \mathrm{Hg})$ & $111 \pm 9.79$ & $112.1 \pm 10.02$ & 0.2 \\
\hline Heart rate (beats/min) & $105.2 \pm 9.18$ & $106.5 \pm 8.5$ & 0.4 \\
\hline $\mathrm{RR}(/ \mathrm{min})$ & $18.9 \pm 2.67$ & $18.95 \pm 2.48$ & 0.3 \\
\hline \multicolumn{4}{|l|}{ Risk factors } \\
\hline Smoking & $13(28 \%)$ & $12(29 \%)$ & 0.32 \\
\hline Immobilization & $11(24 \%)$ & $11(26 \%)$ & 0.22 \\
\hline Surgery/major trauma in 1 month & $7(16 \%)$ & $6(14 \%)$ & 0.5 \\
\hline Diabetes mellitus & $5(11 \%)$ & $4(10 \%)$ & 0.34 \\
\hline Dyslipidemia & $5(11 \%)$ & $4(10 \%)$ & 0.6 \\
\hline Active malignancy & $1(2 \%)$ & $1(2.5 \%)$ & 0.19 \\
\hline OCP/estrogen use & $0(0 \%)$ & $1(2.5 \%)$ & 0.7 \\
\hline \multicolumn{4}{|l|}{ Presenting symptom } \\
\hline Dyspnea & $35(79 \%)$ & $32(78 \%)$ & 0.11 \\
\hline Chest pain & $25(56 \%)$ & $22(53 \%)$ & 0.32 \\
\hline Syncope & $3(7 \%)$ & $2(4.8 \%)$ & 0.5 \\
\hline Duration of illness (days) & $3.65 \pm 2.25$ & $3.3 \pm 1.83$ & 0.6 \\
\hline
\end{tabular}

BMI: body mass index; RR: respiratory rate; SBP: systolic blood pressure; OCP: oral contraceptive pill.

history of surgery or major trauma with in past 1 month in $15 \%(\mathrm{n}=13)$, dyslipidemia in $10 \%(\mathrm{n}=9)$, diabetes mellitus in $10 \%(\mathrm{n}=9)$, active malignancy in $2.5 \%(\mathrm{n}=2)$, and oral contraceptive/estrogen use in $1 \%(n=1)$ of patients, which were not significantly different in both groups. Dyspnea was the most common symptom in $80 \%(\mathrm{n}=69)$ of all patients, followed by chest pain $(55 \%, n=47)$ and syncope $(6 \%, n=$ 5) (Table 1).

\section{Baseline diagnostic evaluation}

All the study patients had documented RV dysfunction on echocardiography. Mean RV/LV size ratio in apical four- chamber view was 1.15 with $1.14 \pm 0.11$ and $1.16 \pm 0.14$ in group I and II, respectively, which were not different significantly. Only four patients in group I and three patients in group II had RV/LV ratio $<0.9$ but had RV dysfunction as evidenced by TAPSE $<16 \mathrm{~mm}$. There was evidence of myocardial injury in all the study patients either in form of elevated troponin I or T. Elevation of troponin T was in $34(75 \%)$ and $28(70 \%)$ patients among group I and group II, respectively, whereas elevated troponin I was found in $25(55 \%)$ and $26(65 \%)$ patients among group I and group II, respectively, though not different significantly. Mean PASP at presentation (calculated by tricuspid regurgitation jet velocity) in the patients was $49.09 \pm 3.02 \mathrm{~mm} \mathrm{Hg}$, with comparable in both groups (group I: $48.90 \pm 3.0$ mm Hg; group II: $49.21 \pm 3.09$ mm Hg; $\mathrm{P}=0.6$ )

Table 2. Diagnostic Workup and Treatment $(n=86)$

\begin{tabular}{llll}
\hline Variables & Group I $($ TNK; $\mathbf{n}=\mathbf{4 5})$ & Group II (placebo; n = 41) & P-value \\
\hline $\mathrm{O}_{2}$ treatment given & $36(80 \%)$ & $30(75 \%)$ & 0.06 \\
RV/LV size ratio & $1.14 \pm 0.11$ & $1.16 \pm 0.14$ & 0.08 \\
Troponin T elevation & $34(75 \%)$ & $28(70 \%)$ & 0.2 \\
Troponin I elevation & $25(55 \%)$ & $26(65 \%)$ & 0.4 \\
Either TropT/tropelevation & $45(100 \%)$ & $41(100 \%)$ & 0.5 \\
Baseline mean PASP, mm Hg & $48.90 \pm 3.0$ & $49.21 \pm 3.09$ & 0.6 \\
UFH given before randomization & $11(25 \%)$ & $14(35 \%)$ & 0.2 \\
\hline
\end{tabular}

$\mathrm{O}_{2}$ : oxygen; RV: right ventricle; LV: left ventricle; PASP: pulmonary artery systolic pressure; UFH: unfractionated heparin. 
Table 3. Efficacy and Safety End Points $(n=86)$

\begin{tabular}{|c|c|c|c|}
\hline Variables & Group I (TNK; $\mathbf{n}=45)$ & Group II (placebo; $n=41)$ & P-value \\
\hline Primary composite outcome within 7 days & $2(4.5 \%)$ & $8(20 \%)$ & 0.04 \\
\hline \multicolumn{4}{|l|}{ Secondary end points } \\
\hline All cause death & $2(4.5 \%)$ & $2(5 \%)$ & 0.3 \\
\hline Hemodynamic decompensation & $2(4.5 \%)$ & $8(20 \%)$ & 0.04 \\
\hline Recurrent PE within 7 days & $2(4.5 \%)$ & $1(2 \%)$ & 0.3 \\
\hline Rehospitalization within 30 days & $2(4.5 \%)$ & $4(10 \%)$ & 0.29 \\
\hline Death within 30 days & $2(4.5 \%)$ & $2(5 \%)$ & 0.18 \\
\hline \multicolumn{4}{|l|}{ Others } \\
\hline Mean PASP at D7 (mm Hg) & $32.80 \pm 4.02$ & $38.13 \pm 4.49$ & 0.04 \\
\hline Mean $\downarrow$ in PASP from baseline $(\mathrm{mm} \mathrm{Hg})$ & $14.10 \pm 3.95$ & $11.08 \pm 4.23$ & 0.003 \\
\hline Improvement in RV function & $31(70 \%)$ & $16(40 \%)$ & 0.001 \\
\hline Need for mechanical ventilation & $2(4.5 \%)$ & $2(5 \%)$ & 0.6 \\
\hline Mean hospital stay (days) & $8.1 \pm 2.51$ & $11.1 \pm 2.14$ & 0.001 \\
\hline \multicolumn{4}{|l|}{ Safety end points (bleeding within D7) } \\
\hline Major bleeding & $1(2 \%)$ & $1(2 \%)$ & 0.45 \\
\hline Minor Bleeding & $7(16 \%)$ & $5(12 \%)$ & 0.04 \\
\hline Hemorrhagic stroke & $1(2 \%)$ & $0(0 \%)$ & 0 \\
\hline
\end{tabular}

PE: pulmonary embolism; PASP: pulmonary artery systolic pressure; RV: right ventricle.

(Table 2).

\section{Efficacy and safety outcome}

Between randomization and D7, the primary efficacy outcome occurred significantly lesser in group I vs. group II $(n=2 ; 4.5 \%$ vs. $\mathrm{n}=8 ; 20 \% ; \mathrm{P}=0.04)$. Two patients died in each group (4.5\% and $5 \% ; \mathrm{P}=0.3)$ but hemodynamic decompensation or collapse was significantly more in group II than group I ( $\mathrm{n}=$ $8 ; 20 \%$ vs. $\mathrm{n}=2 ; 4.5 \% ; \mathrm{P}=0.04$ ). Recurrent $\mathrm{PE}$ within 7 days in both groups was not different significantly (group I: $\mathrm{n}=2$; $4.5 \%$ vs. group II: $n=1 ; 2 \% ; P=0.3)$. Two patients $(4.5 \%)$ in group I were rehospitalized within 30 days as compared to four patients $(10 \%)$ in group II but difference was statistically not significant $(\mathrm{P}=0.29)$. Reasons for rehospitalization were pneumonia with chest pain and fever for evaluation. Two deaths occurred in group I (4.5\%) and group II (5\%), respectively which were statistically not significant $(P=0.18)$. All mortalities occurred within first 7 days and there was no death between D7 and 30 days. Sepsis and hemodynamic collapse were the causes of deaths in group I and group II, respectively. At D7, mean PASP decreased significantly in both groups, i.e., from $48.90 \pm$ 3.0 to $32.80 \pm 4.02 \mathrm{~mm} \mathrm{Hg}$ in group I and from $49.21 \pm 3.09$ to $38.13 \pm 4.49 \mathrm{~mm} \mathrm{Hg}$ in group II. At D7, mean PASP fall was $28.8 \%(14.1 \mathrm{~mm} \mathrm{Hg})$ in group I and by $22.5 \%(11.08 \mathrm{~mm} \mathrm{Hg})$ in group II which were significant $(\mathrm{P}=0.03)$. Improvement in RV function was significantly greater in group I than group II ( $\mathrm{n}=31 ; 70 \%$ vs. $\mathrm{n}=16 ; 40 \% ; \mathrm{P}=0.001)$. Two patients required mechanical ventilation in group I $(4.5 \%)$ and group II $(5 \%)$, respectively, which were not different. These four patients lat- er succumbed to death. Mean hospital stay was significantly shorter in group I: $8.1 \pm 2.51$ days compared to $11.1 \pm 2.14$ days in group II $(\mathrm{P}=0.001)$. Major reason for this prolonged hospitalization was hemodynamic decompensation (Table 3 ).

\section{Safety outcomes}

Major bleeding occurred between randomization and D7 in one patient $(2 \%)$ in group I and one patient in group II $(2.5 \%)$ which were not different. Patient in group I had intracranial bleed detected on fourth day while that in group II had lower gastrointestinal bleeding requiring 4 Unit of blood transfusion. Minor bleeding was more in group I than group II $(\mathrm{n}=7 ; 16 \%$ vs. $\mathrm{n}=5 ; 12 \% ; \mathrm{P}=0.04)$.

\section{Discussion}

Submassive PE is defined by evidence of RV dysfunction and/ or evidence of myocardial necrosis in absence of hemodynamic instability in patients of acute PE. A review of randomized trials indicated that thrombolysis may be associated with a reduction in mortality or recurrent PE in high-risk patients who present with hemodynamic instability. Clinical trials of thrombolysis in acute submassive PE have demonstrated more rapid, immediate hemodynamic improvement and clot resolution following thrombolysis, but unclear mortality benefits and therefore remains controversial [8,9]. Miller et al [10] showed that streptokinase infusion over $72 \mathrm{~h}$ resulted in a significant reduction of SPAP, total pulmonary resistance, and 
the angiographic index of PE severity. In comparison, conventional heparin anticoagulation had no appreciable effect on the above parameters during the first 3 days. Goldhaber et al [11] compared alteplase (100 $\mathrm{mg}$ infusions over $2 \mathrm{~h}$ ) to heparin alone among 101 patients based on echocardiographic indicators of RV pressure overload and dysfunction in the evaluation of PE severity. But it did not exclude patients with a normally functioning right ventricle on echocardiography. Therefore, particular focus on the patient population with submassive PE was lacking. They showed rapid improvement of RV function in the alteplase group as assessed by 24-h echocardiographic follow-up. However, thrombolysis was not shown to have any effect on survival, partly due to the low $(2 \%)$ overall in-hospital mortality. No clinical episodes of recurrent PE were noted among rt-PA patients, but there were two fatal and three non-fatal clinically suspected recurrent PEs within 14 days in patients randomized to heparin alone. Konstantinides et al [8] in MAPPET-3 trial (The Management Strategies and Prognosis of Pulmonary Embolism Trial-3) randomized 256 patients with submassive PE to receive recombinant tissue plasminogen activator (tPA) $100 \mathrm{mg}$ over a 2-h period followed by UFH infusion or placebo plus heparin anticoagulation. Compared with heparin anticoagulation alone, tPA resulted in a significant reduction in primary study endpoint (escalation of therapy defined as catecholamine infusion, rescue fibrinolysis, mechanical ventilation, cardiopulmonary resuscitation, or emergency surgical embolectomy). However, there was difference in mortality among both the groups. In accordance with the studies conducted by Miller et al [10] and Goldhaber et al [11], our study also showed significant reduction in PASP and improvement in RV function with thrombolytic therapy as compared to heparin group. Mean PASP decreased significantly in both groups but decrease in mean PASP at D7 was more in TNK than heparin group. Similarly, more improvement in RV function was seen in patients who received TNK than those receiving heparin. In contrast to MAPPET-3 trial, our study did not show any significant reduction in requirement for mechanical ventilation with thrombolytic therapy. Becattini et al [12] in TIPES study which evaluated the effect of TNK on RV dysfunction assessed by echocardiography in hemodynamically stable patients with submassive PE showed that TNK had a significant reduction in $\mathrm{RV}$ dysfunction with no significant reduction in all cause mortality at 30 days, which is similar to our findings. Sharifi et al [13] in MOPETT Trial (moderate pulmonary embolism treated with thrombolysis) of 121 patients with moderate $\mathrm{PE}$ randomized to receive a "safe dose" alteplase plus anticoagulation or anticoagulation alone. There was significant reduction in duration of hospitalization, bleeding during the index hospitalization, recurrence of $\mathrm{PE}$ and combination of death plus PE recurrence. Despite a positive trend in favor of a "safe dose" thrombolysis, no significant difference was noted in death rate. We did not find any difference in mortality in both groups, probably because overall mortality rate was low (4.6\%). Rate of rehospitalization within 30 days and recurrent $\mathrm{PE}$ also did not differ significantly in both the groups $(\mathrm{P}>$ 0.05). Similar to MOPPET Trial, mean hospital stay was significantly reduced with thrombolytic therapy as compared to heparin only group. PEITHO trial [13] (pulmonary embolism thrombolysis) which randomized 1,006 patients with intermediate-risk (submassive) PE to either systemic thrombolysis with weight adjusted TNK or to placebo, on a background of weight-based heparin. The primary composite endpoint of allcause mortality or hemodynamic decompensation occurred in $2.6 \%$ of patients in thrombolysis arm compared to $5.6 \%$ of patients in the placebo group which was mostly driven by a decreased occurrence of hemodynamic instability although 30-day mortality alone did not differ between the groups. A meta-analysis which pooled data from eight trials containing 1,775 patients with intermediate-risk PE showed that thrombolysis was associated with lower all-cause mortality (OR: 0.48 ; 95\% CI: $0.25-0.92)$, but significantly more bleeding events (OR: 3.19; 95\% CI: 2.07 - 4.92) [14]. In our study, similar findings were noted as in PEITHO trial [13] though hemodynamic instability was more in our group in placebo arm. In PEITHO trial [13], there was significantly increased risk of extracranial bleeding with thrombolysis as compared to heparin group $(6.3 \%$ vs. $1.2 \%)$ and intracerebral hemorrhage $(2.4 \%$ vs. $0.2 \%)$. In our study, there was no significant difference in major bleeding in both the groups as intracranial bleed was more in thrombolytic arm whereas extracranial bleed was more in placebo arm. Another finding was significantly increased the risk of minor bleeding in thrombolytic arm than placebo. Risk of stroke that might be a concern with thrombolysis did not differ in both groups.

\section{Limitations}

This study represents a single-center experience with limited number of patients though baseline demographic and clinical characteristics appear to be well matched.

\section{Conclusion}

Patients with acute submassive PE do not derive overall mortality benefit, recurrent $\mathrm{PE}$ and rehospitalization with thrombolytic therapy but had improved clinical course in form of decrease in hemodynamic decompensation, mean hospital stay, PASP and improvement of RV function at the cost of increased minor bleeding.

\section{Conflicts of Interest}

None.

\section{Financial Disclosure}

None.

\section{References}

1. Goldhaber SZ, Visani L, De Rosa M. Acute pulmonary 
embolism: clinical outcomes in the International Cooperative Pulmonary Embolism Registry (ICOPER). Lancet. 1999;353(9162):1386-1389.

2. Fanikos J, Piazza G, Zayaruzny M, Goldhaber SZ. Longterm complications of medical patients with hospitalacquired venous thromboembolism. Thromb Haemost. 2009;102(4):688-693.

3. Mehta NJ, Jani K, Khan IA. Clinical usefulness and prognostic value of elevated cardiac troponin I levels in acute pulmonary embolism. Am Heart J. 2003;145(5):821-825.

4. Pacouret G, Schellenberg F, Hamel E, Charbonnier B, Mouray H. [Troponin I in massive acute pulmonary embolism: results of a prospective series]. Presse Med. 1998;27(32):1627.

5. Grifoni S, Olivotto I, Cecchini P, Pieralli F, Camaiti A, Santoro G, Conti A, et al. Short-term clinical outcome of patients with acute pulmonary embolism, normal blood pressure, and echocardiographic right ventricular dysfunction. Circulation. 2000;101(24):2817-2822.

6. Schulman S, Kearon C. Definition of major bleeding in clinical investigations of antihemostatic medicinal products in non-surgical patients. J Thromb Haemost. 2005;3(4):692-694.

7. Long B, Koyfman A. Current Controversies in Thrombolytic Use in Acute Pulmonary Embolism. J Emerg Med. 2016;51(1):37-44.

8. Konstantinides S, Geibel A, Heusel G, Heinrich F, Kasper W. Heparin plus alteplase compared with heparin alone in patients with submassive pulmonary embolism. N Engl J Med. 2002;347(15):1143-1150.

9. Fasullo S, Scalzo S, Maringhini G, Ganci F, Cannizzaro S, Basile I, Cangemi D, et al. Six-month echocardiographic study in patients with submassive pulmonary embolism and right ventricle dysfunction: comparison of thrombolysis with heparin. Am J Med Sci. 2011;341(1):33-39.

10. Miller GA, Sutton GC, Kerr IH, Gibson RV, Honey M. Comparison of streptokinase and heparin in treatment of isolated acute massive pulmonary embolism. Br Heart J. 1971;33(4):616.

11. Goldhaber SZ, Haire WD, Feldstein ML, Miller M, Toltzis R, Smith JL, Taveira da Silva AM, et al. Alteplase versus heparin in acute pulmonary embolism: randomised trial assessing right-ventricular function and pulmonary perfusion. Lancet. 1993;341(8844):507-511.

12. Becattini C, Vedovati MC, Agnelli G. Prognostic value of troponins in acute pulmonary embolism: a meta-analysis. Circulation. 2007;116(4):427-433.

13. Sharifi M, Bay C, Skrocki L, Rahimi F, Mehdipour M. Moderate pulmonary embolism treated with thrombolysis (from the "MOPETT" Trial). Am J Cardiol. 2013;111(2):273-277.

14. Chatterjee S, Chakraborty A, Weinberg I, Kadakia M, Wilensky RL, Sardar P, Kumbhani DJ, et al. Thrombolysis for pulmonary embolism and risk of all-cause mortality, major bleeding, and intracranial hemorrhage: a metaanalysis. JAMA. 2014;311(23):2414-2421. 